\title{
TRIANGULARIZABLE ALGEBRAS OF COMPACT OPERATORS
}

\author{
G. J. MURPHY
}

AbStract. It is shown that a closed algebra $A$ of compact operators is triangularizable if and only if the algebra $A / \operatorname{rad}(A)$ is commutative.

An algebra $A$ of operators on a Banach space $X$ is triangularizable if there exists a maximal chain of subspaces of $X$ invariant for $A$ [7]. If $X$ is finite dimensional, this reduces to the usual concept, since in this case $A$ is triangularizable if and only if there exists a basis for $X$ with respect to which all the operators in $A$ have upper triangular matrices. Recently the concept of triangularizability has attracted some attention; see $[2,6,7]$. Also, for some earlier finite dimensional results see McCoy $[9,10]$. In this paper we show that if $A$ is a closed algebra of compact operators, then $A$ is triangularizable if and only if $A / \operatorname{rad}(A)$ is commutative (where $\operatorname{rad}(A)$ denotes the Jacobson radical of $A$ ). It follows that if $A$ is radical or commutative then $A$ is necessarily triangularizable.

Notation. $X$ will always denote a complex Banach space, $\mathscr{B}(X)$ the algebra of bounded linear operators on $X$, and $\mathcal{K}(X)$ the ideal of compact operators. The spectrum and spectral radius are denoted by $\sigma$ and $r$ respectively. Subspaces of $X$ are always assumed to be closed. $A$ always denotes a complex Banach algebra. If $A \subseteq \mathscr{B}(X)$, a subspace $M$ of $X$ is invariant for $A$ if $T(M) \subseteq M(T \in A)$. Finally if $S, T \in \mathscr{B}(X),[S, T]$ denotes their commutator, $[S, T]=S T-T S$.

Defintion. A closed subalgebra $A$ of $\mathscr{B}(X)$ is triangularizable if there exists a maximal chain of subspaces of $X$ each of which is invariant for $A$.

Before stating the next lemma we need to establish some notation. Let $T \in$ $\mathscr{B}(X)$, and $\mathscr{F}$ a maximal chain of subspaces, each invariant for $T$. If $M \in \mathcal{F}$ and $M_{-} \neq M$, then we can choose $z \in M$ so that $M_{-} \oplus[z]=M$, where [z] denotes the span of $z$. Hence $T z=y+\alpha z$ for some $y \in M_{-}$and for some complex number $\alpha$. It is easily seen $\alpha$ does not depend on $z$, and we write $\alpha=\alpha_{M}(T)$. If $M_{-}=M$, we put $\alpha_{M}(T)=0 .\left(M_{-}=\cup\{N \in \mathscr{F}: N \subset M\}\right.$. See [7].)

LEMMA 1. Let $T \in \mathcal{K}(X)$ and suppose $\mathscr{F}$ is a maximal chain of subspaces of $X$, all invariant for $T$. Then $r(T)=\sup \left\{\left|\alpha_{M}(T)\right|: M \in \mathscr{F}\right\}$.

Proof. Ringrose [11] proves that $\sigma(T) \backslash\{0\}=\left\{\alpha_{M}(T): M \in \mathcal{F}\right\} \backslash\{0\}$, from which the above result is immediate.

Received by the editors November 18, 1980 and, in revised form, June 9, 1981.

1980 Mathematics Subject Classification. Primary 47D30. 
THEOREM 1. Let $A$ be a closed algebra of compact operators on a Banach space $X$. Then the following are equivalent.

(i) $A$ is triangularizable.

(ii) $r(S+T)<r(S)+r(T)(S, T \in A)$.

(iii) $A / \operatorname{rad} A$ is commutative.

Proof. The equivalence of (ii) and (iii) holds for any Banach algebra and is due to Aupetit [1, p. 48].

To show (i) implies (ii), suppose $A$ is triangularizable, and let $\mathcal{F}$ be a maximal chain of subspaces of $X$, all of which are invariant for $A$. If $S, T \in A$ and $M \in \mathcal{F}$ then it is easily seen that $\alpha_{M}(S+T)=\alpha_{M}(S)+\alpha_{M}(T)$. Thus

$$
\begin{aligned}
r(S+T) & =\sup _{M \in \mathcal{F}}\left|\alpha_{M}(S+T)\right| \quad \text { (by Lemma 1) } \\
& <\sup _{M \in \mathcal{F}}\left|\alpha_{M}(S)\right|+\sup _{M \in \mathscr{F}}\left|\alpha_{M}(T)\right|=r(S)+r(T) .
\end{aligned}
$$

Finally to show (iii) implies (i), suppose $A / \operatorname{rad} A$ is commutative. Then for all $S, T, U$ in $A, S(T U-U T)$ is quasinilpotent. Thus $P(T, U)[T, U]$ is quasinilpotent for every polynomial $P\left(X_{1}, X_{2}\right)$ in two noncommuting variables. Hence by Corollary 3.5 of [7], $A$ is triangularizable.

COROLLARY. Every closed radical subalgebra of $\mathcal{K}(X)$ is triangularizable.

COROLlARY [7]. Every closed commutative subalgebra of $\mathcal{K}(X)$ is triangularizable.

Corollary [7]. If $S, T \in \mathcal{K}(X)$ and $[S, T]$ commutes with $S$ and $T$, then the closed algebra $A$ generated by $S$ and $T$ is triangularizable.

Proof. By a theorem of Kleinecke and Shirokov (see [5, p. 129]), $S[S, T]=$ $[S, T] S$ implies $r([S, T])=0$. Also, if $U \in A$, then $[S, T]$ and $U$ commute, so $r(U[S, T]) \leqslant r(U) r([S, T])=0$. Thus $[S, T] \in \operatorname{rad} A$, and so $A / \operatorname{rad} A$ is commutative.

REMARKs. (i) We will now give an example of a Banach space $X$ and a closed subalgebra $A$ of $\mathscr{B}(X)$ which is triangularizable, but for which $A / \operatorname{rad} A$ is not commutative. This is a partial answer to questions in Remark 5.2 of [7].

Let $X$ be the separable Hilbert space $l^{2}$, with the canonical basis $\left(e_{n}\right)_{n-1}^{\infty}$. Let $U$ be the unilateral shift, and $W$ the shift with weights $(-1)^{n}$, so $U e_{n}=e_{n+1}$ and $W e_{n}=(-1)^{n} e_{n+1}$. Let $A$ be the closed subalgebra of $\mathscr{B}(X)$ generated by $U$ and $W$. $A$ is clearly triangularizable (take $\mathscr{F}=\left\{M_{n}: n=0,1,2, \ldots, \infty\right\}$ where $M_{0}=0$, $M_{\infty}=X$ and $M_{n}$ is the closed span of $\left.\left\{e_{n}, e_{n+1}, \ldots\right\}(n=1,2,3, \ldots)\right)$. Now $[U, W] e_{n}=2(-1)^{n} e_{n+2}$, so $[U, W]^{n} e_{1}=(-2)^{n} e_{2 n-1}(n=1,2, \ldots)$. Thus $\left\|[U, W]^{n}\right\|$ $>2^{n}$, implying $r([U, W]) \geqslant 2$. Therefore $[U, W] \notin \operatorname{rad} A$, and so $A / \operatorname{rad} A$ is not commutative.

(ii) The example of Enflo [4] of a Banach space $X$ of infinite dimension and an operator $T$ on $X$ having only trivial invariant subspaces would imply that commutative algebras are not necessarily triangularizable (consider the closed subalgebra of $\mathscr{B}(X)$ generated by $T$ above). 


\section{G. J. MURPHY}

\section{REFERENCES}

1. B. Aupetit, Propriétés spectrales des algèbres de Banach, Lecture Notes in Math., Springer-Verlag, Berlin and New York, 1979.

2. M.-D. Choi, C. Laurie and H. Radjavi, On commutators and invariant subspaces, Linear and Multilinear Algebra 9 (1981), 329-340.

3. H. Dowson, Spectral theory of linear operators, Academic Press, London, 1978.

4. P. Enflo, On the invariant subspace problem in Banach spaces, Séminaire Maurey-Schwartz (1975-1976), Espaces $L^{p}$ Applications Radonifiantes et Géométrie des Espaces de Banach, Exp. No. 14-15, Centre Math., École Polytech., Palaiseau, 1976.

5. P. R. Halmos, A Hilbert space problem book, Van Nostrand, Princeton, N.J., 1967.

6. T. J. Laffey, Simultaneous triangularization of matrices-low rank cases and the nonderogatory case, Linear and Multilinear Algebra 6 (1978), 269-305.

7. C. Laurie, E. Nordgren, H. Radjavi and P. Rosenthal, On triangularization of algebras of operators, preprint.

8. V. Lomonosov, Invariant subspaces for operators commuting with compact operators, Funkcional. Anal. i Priložen. 7 (1973), 55-56 = Functional Anal. Appl. 7 (1973), 213-214.

9. N. H. McCoy, On quasi-commutative matrices, Trans. Amer. Math. Soc. 36 (1934), 327-340.

10. On the characteristic roots of matrix polynomials, Bull. Amer. Math. Soc. 42 (1963), $592-600$.

11. J. R. Ringrose, Super-diagonal forms for compact linear operators, Proc. London Math. Soc. (3) 12 (1962), 367-384.

Department of Mathematics, Dalhousie University, Halifax, Nova Scotia, Canada 